\title{
The Application of the Probabilistic Collocation Method to a Transonic Axial Flow Compressor
}

\author{
G.J.A. Loeven*† and H. Bij ${ }^{\ddagger}$ \\ Delft University of Technology, The Netherlands
}

\begin{abstract}
In this paper the Probabilistic Collocation method is used for uncertainty quantification of operational uncertainties in a transonic axial flow compressor (i.e. NASA Rotor 37). Compressor rotors are components of a gas turbine that are highly sensitive to operational and geometrical uncertainties. Validation of the Probabilistic Collocation method with a Monte Carlo simulation using 10,000 Latin Hypercube samples demonstrated that the Probabilistic Collocation method can successfully be applied to a turbomachinery case. The flow through the rotor is characterized by a bow shock in front of the leading edge, which interacts with the boundary layer of the next blade. The total pressure profile at the inlet of the rotor is assumed to be uncertain. A symmetric beta distribution was used for the pressure profile, with the standard deviation such that the uncertainty is in the same order of the measurement accuracy reported in literature. The mass flow was shown to be the most sensitive to the uncertainty, while the efficiency is least affected. It was shown by the compressor maps that is important to take the uncertainty in the total pressure profile at the inlet into account. The standard deviation of the static pressure field showed that the largest variation is present near the shock wave and mainly in the region of the strongest shock, which is near the tip of the blade.
\end{abstract}

\section{Introduction}

The compressor is one of the most sensitive components of a gas turbine. The performance is sensitive to variability in geometry and operating conditions. Uncertainty analysis is, therefore, of great importance to assure robustness of the design. The goal of this chapter is to demonstrate the use of the Probabilistic Collocation method for a turbomachinery case. Furthermore, the effect of operational uncertainties on the performance of the rotor is investigated. Recently, uncertainty quantification has been applied to CFD computations in turbomachinery. ${ }^{1,2}$ A commonly used test case for turbomachinery is NASA Rotor $37 .{ }^{3,4}$

In 1993, a large group of researchers computed the NASA Rotor 37 test case on invitation of the ASME Turbomachinery Committee using RANS codes. ${ }^{5}$ These were blind simulations, only the geometric data were provided. The contributers to a later study of Dunham ${ }^{4}$ had access to the experimental data when the computations were performed. The comparison between the experiments and CFD computations showed quite some differences. For example the efficiency was almost always underpredicted, possibly due to the treatment of the tip clearance in the simulations. Furthermore, the shock wave is stronger and more upstream in the computations than in the experiments. Dunham ${ }^{4}$ states that the main contribution to the differences lies in the assumption of steady flow and the choice of turbulence model.

Gopinathrao, Bagshaw, Mabilat and Alizadeh² have successfully applied the Probabilistic Collocation method to NASA Rotor 37 using Fluent and investigated how the choice of probability distribution for the uncertain parameters affects the solution. In this chapter a quasi-2D study is used to validate the applicability of the Probabilistic Collocation method to a turbomachinery test case against a Monte Carlo simulation. Furthermore, standard deviation plots of flow quantities are shown to reveal areas in the flow field that are sensitive to operational uncertainties.

The rotor of a gas turbine is subject to several sources of uncertainty. One of them is operational uncertainty. It is always present when the rotor is operated in an environment that is different from the

\footnotetext{
*Corresponding author, email: G.J.A.Loeven@TUDelft.nl

${ }^{\dagger}$ Researcher, Department of Aerospace Engineering, P.O. Box 5058, 2600 GB Delft, The Netherlands, member AIAA

${ }^{\ddagger}$ Full Professor, Department of Aerospace Engineering, P.O. Box 5058, 2600 GB Delft, The Netherlands, member AIAA.
} 
design conditions. Two operational uncertainties are considered. First the static outlet pressure in the validation test case and secondly, the total pressure profile at the inlet of the rotor for the 3D simulation. Another source of uncertainty is geometrical imperfection. Due to manufacturing tolerances or wear, the actual geometry might be different from the designed geometry. Especially, the effect of the tip clearance is known to have a significant effect on the efficiency of the rotor. ${ }^{6,7,8}$ In this chapter operational uncertainties are considered.

The uncertainties in the NASA Rotor 37 test case are propagated using the Probabilistic Collocation $\operatorname{method}^{9,10,11,12}$ with the FINE/Hexa solver of Numeca International as a deterministic flow solver. First a quasi-2D computation, which represents a slice at $50 \%$ blade height, is performed to validate the applicability of the Probabilistic Collocation to the NASA Rotor 37 test case. A Monte Carlo simulation of 10,000 Latin Hypercube samples has been performed to obtain a stochastic reference solution. The quasi-2D case contains all features that characterize the flow through the compressor rotor. One important feature is the bow shock in front of the leading edge of the blade. The shock wave impinges on the boundary layer on the next blade. The static outlet pressure is assumed to be uncertain and to have a symmetric beta probability distribution. A symmetric beta distribution has the property that the mean value is most probable to occur. Furthermore, it is limited to an interval, where the probability smoothly decreases to zero near the edges of the interval. The Probabilistic Collocation method shows with 5 deterministic solves good correspondence with the Monte Carlo simulation.

After validating the Probabilistic Collocation method, it is applied to a $3 \mathrm{D}$ simulation of the rotor. Uncertainties are present in the total pressure profile at the inlet of the rotor. A symmetric beta distribution is assumed, with the interval chosen such that the standard deviation is in the order of the accuracy of the experiments of Reid and Moore. ${ }^{3}$ The effect on the efficiency, total pressure ratio, mass flow and the compressor map are shown. Furthermore, the mean and standard deviation of the static pressure in the flow field are visualized to show areas in the flow field that are sensitive to the uncertainties in the total pressure profile at the inlet of the rotor.

\section{Deterministic test case description}

NASA Rotor 37 was originally designed and tested as part of a research program involving four related axial-flow compressor stages ${ }^{3}$ typical for aircraft turbine engines. It is shown in figure 1(a). The rotor has 36 blades with an aspect ratio of 1.19 . The designed efficiency is $\eta_{\text {design }}=0.877$ with a total pressure ratio of $p_{2} / p_{1 \text { design }}=2.106$. The measured peak efficiency by Reid and Moore ${ }^{3}$ was $\eta_{\text {measured }}=0.876$ with a total pressure ratio of $p_{2} / p_{1_{\text {measured }}}=2.056$. The maximum attained mass flow is $\dot{m}_{\text {choke }}=20.93 \mathrm{~kg} / \mathrm{s.}^{4}$

\section{A. Computational settings}

The flow through NASA Rotor 37 is simulated by the Reynolds averaged Navier-Stokes equations in combination with the Spalart-Allmaras turbulence model. The equations are solved on a computational grid, which is described in the next section. The boundary conditions are treated at the end of this section.

\section{The computational model and grid}

The computational model of the NASA Rotor 37 consists of one blade with periodic boundary conditions, shown in figure 1(d). The model of the complete rotor, e.g. 36 repeated blades, is shown in front view in figure $1(\mathrm{~b})$ and from the side in $1(\mathrm{c})$.

The blade geometry can be found in the work of Reid and Moore. ${ }^{3}$ Between the blade and the shroud a small gap is present, known as the tip clearance. The tip clearance is equal to $0.356 \mathrm{~mm}$. It is important to model the tip clearance correctly, since it has a significant influence on the results. Extensive research has been performed to the effect of the tip clearance by Gerolymos and Vallet ${ }^{7}$ and Beheshti et al. ${ }^{8}$

The influence of the 3D computational grid was investigated by Tartinville and Hirsch. ${ }^{13}$ The grid that is used, contains approximately 600,000 cells. Parts of the grid are shown in figure 2. A top view of the surface grid on the hub is displayed in figure 2(a). Figure 2(b) shows a close view of the blade near the hub. It is assured that along the surface $y^{+}=1-2$, to comply with the Spalart-Allmaras turbulence model. Furthermore, the grid is sufficiently refined near the tip to capture the flow phenomena around the tip clearance. 


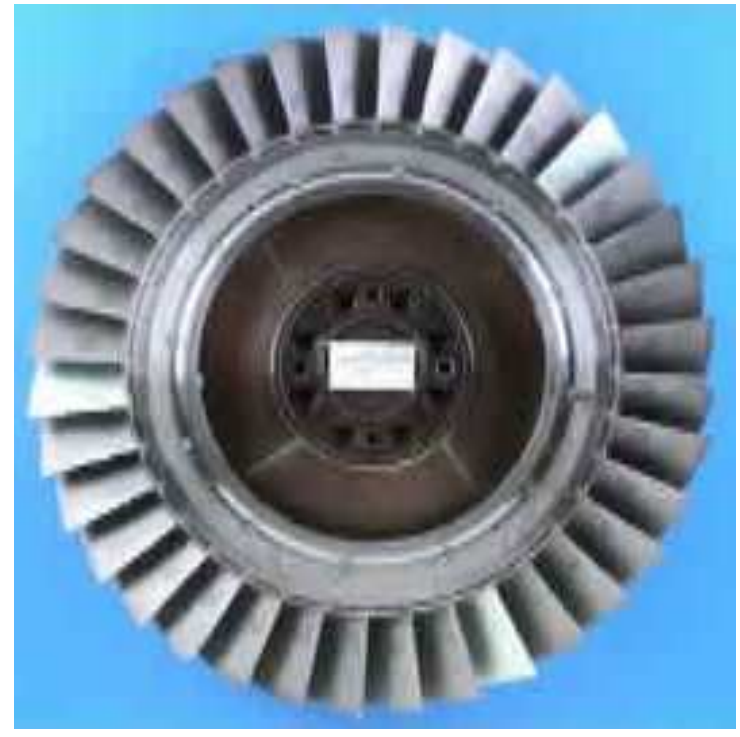

(a)

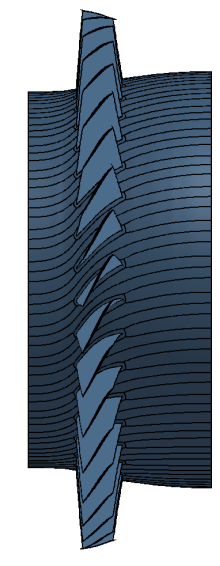

(c)

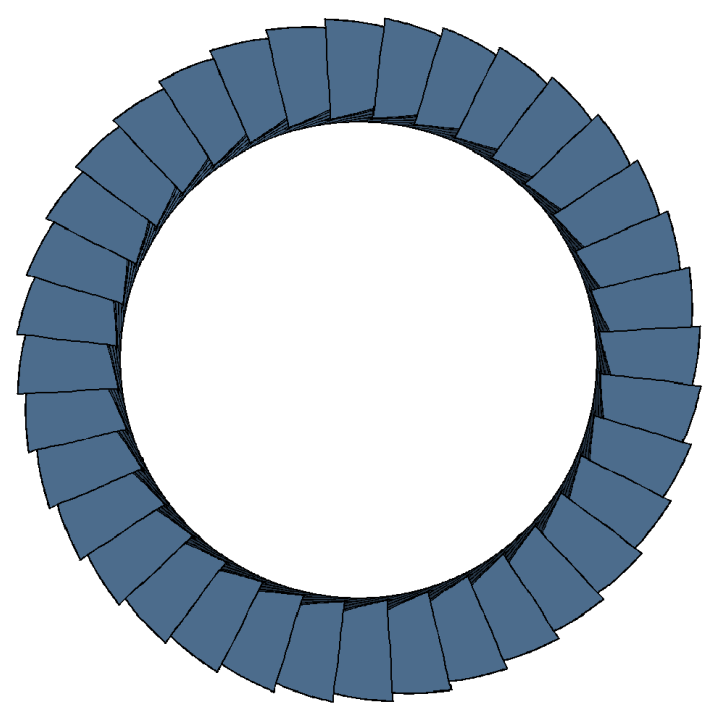

(b)

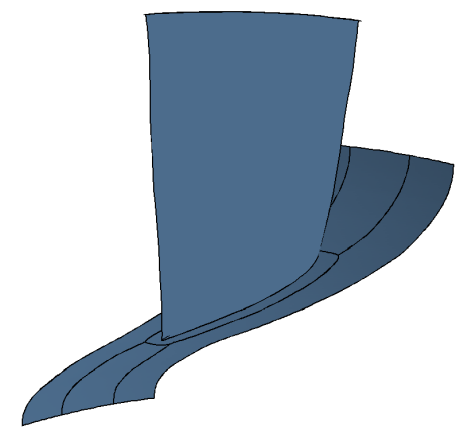

(d)

Figure 1. NASA Rotor 37, (a) real model front [Source: http://www.grc.nasa.gov] and (b) computational model front, (c) model side, (d) single blade. 


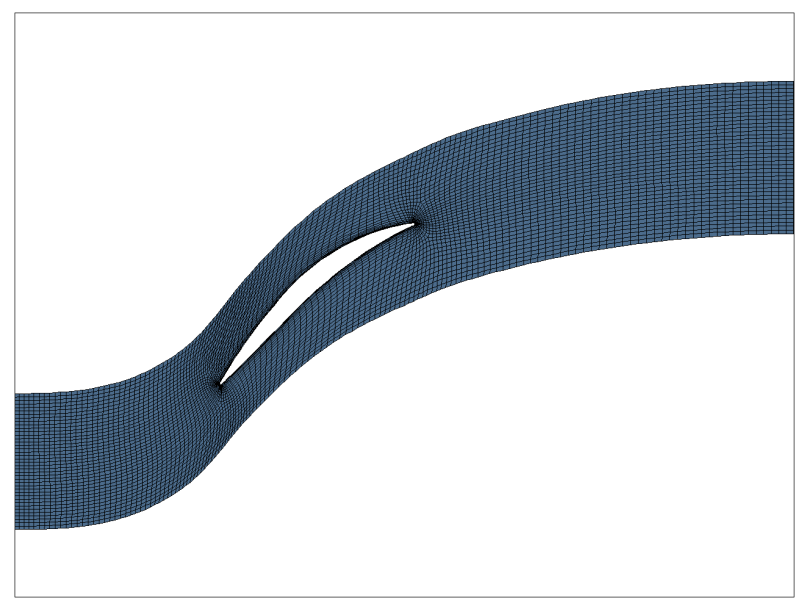

(a)

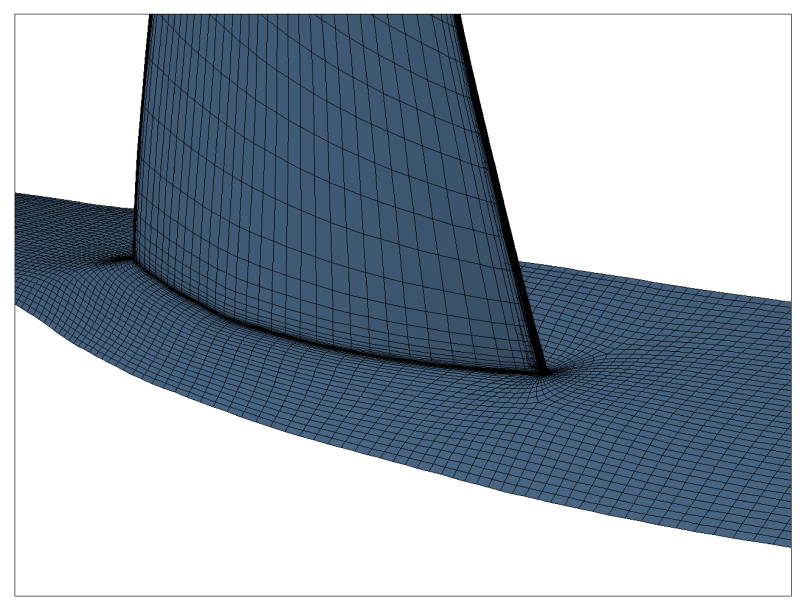

(b)

Figure 2. 3D computational grid for NASA Rotor 37, (a) a top view of the grid on the hub and (b) a close view of the blade near the hub.

\section{Boundary conditions}

The rotational speed of the rotor is $17188.7 \mathrm{rpm}(1800 \mathrm{rad} / \mathrm{s})$, leading to a tip-speed of $454 \mathrm{~m} / \mathrm{s}(1500 \mathrm{ft} / \mathrm{s})$. The hub and blade in the computational model are rotating, while the shroud is kept fixed. At the inlet, a total pressure and total temperature profile are specified, which are experimentally determined and can be found in the work of Dunham. ${ }^{4}$ The static outlet pressure is varied to change the mass flow and construct the compressor maps. At maximal efficiency, the static outlet pressure is set to 1.175 bar.

\section{B. Deterministic solution}

The maximum adiabatic efficiency of the rotor is found to be $\eta=0.867$. At the maximum efficiency, the mass flow is $\dot{m}=20.737 \mathrm{~kg} / \mathrm{s}$ and the total pressure ratio is $p_{2} / p_{1}=2.077$. The maximum mass flow is found to be $\dot{m}_{\text {choke }}=20.903 \mathrm{~kg} / \mathrm{s}$. The results are summarized in table 1 together with the experimental values measured by Reid and Moore. ${ }^{3}$ All values are within $1.03 \%$ of the measured data.

Table 1. Numerical simulation and experimental results ${ }^{3}$ for NASA Rotor 37.

\begin{tabular}{ccc}
\hline Quantity & Experimental & Numerical \\
\hline Maximum adiabatic efficiency $\eta[-]$ & 0.876 & $0.867(-1.03 \%)$ \\
Pressure ratio at max. efficiency $p_{2} / p_{1}[-]$ & 2.056 & $2.077(+1.02 \%)$ \\
Massflow at max. efficiency $\dot{m}[\mathrm{~kg} / \mathrm{s}]$ & 20.74 & $20.737(-0.014 \%)$ \\
Maximum mass flow $\dot{m}_{\text {choke }}[\mathrm{kg} / \mathrm{s}]$ & 20.93 & $20.903(-0.129 \%)$ \\
\hline
\end{tabular}

Figure 3 shows the compressor maps. The figure includes the numerical results and the experimental results from Reid and Moore. ${ }^{3}$ A good agreement between the results is obtained. The only major difference between the numerical simulations and the experiments is the rotor stall (the most left data point in figure 3 ). At stall the mass flow differs $0.8 \mathrm{~kg} / \mathrm{s}(\approx 4 \%)$, which is a large difference. For the main goal, performing an uncertainty analysis for NASA Rotor 37 , it is no problem.

\section{Flow field}

Figure 4 shows the static pressure on the surface of the hub and upper (a) and lower (b) side of the blade. Since the tip velocity is much higher than the root velocity, the shock angle and strength vary along the blade. The static pressure on both sides of the blade shows that the flow has a complex three dimensional structure. 


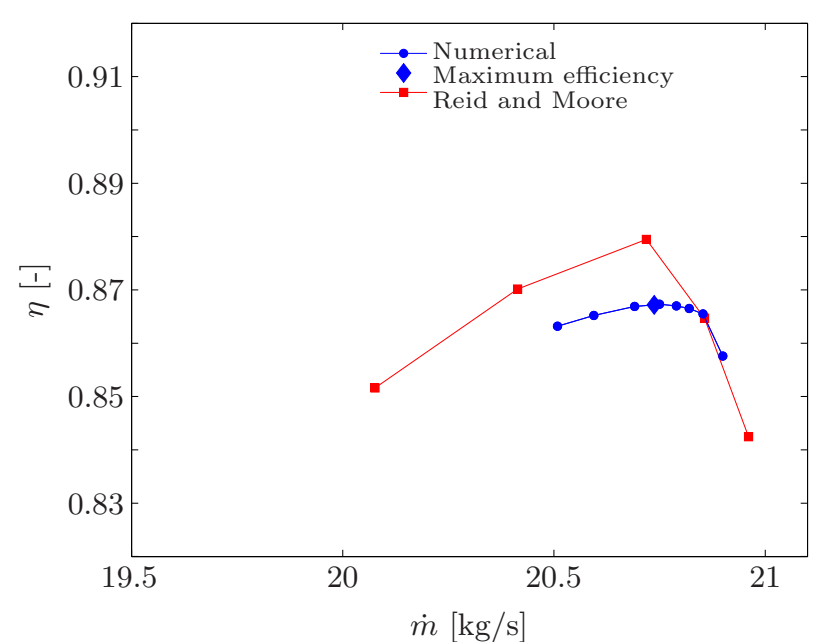

(a)

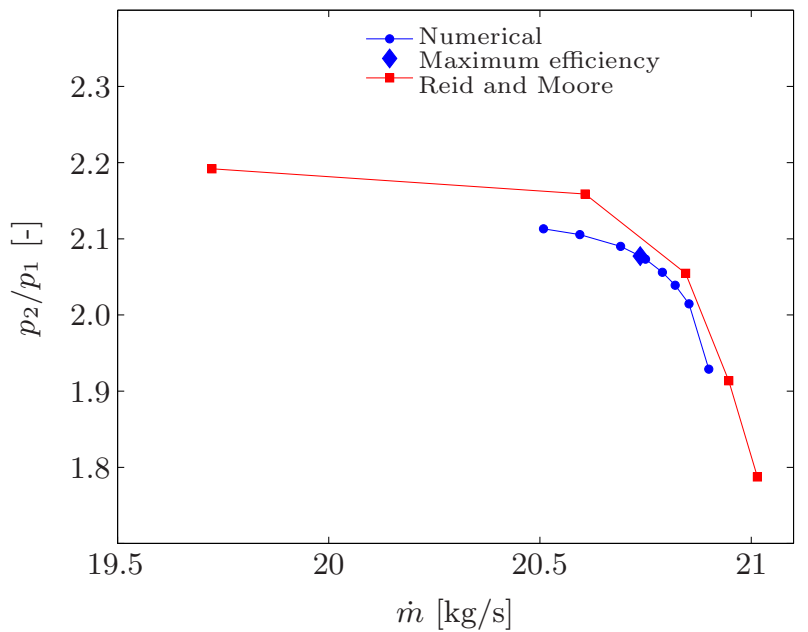

(b)

Figure 3. Compressor maps of NASA Rotor 37, with (a) the adiabatic efficiency $\eta_{\text {adiabatic }}$ and (b) the total pressure ratio $p_{2} / p_{1}$ against the mass flow $\dot{m}$. The figure shows the numerical results, the numerically determined maximum adiabatic efficiency and the experimental results from Reid and Moore ${ }^{3}$.

To obtain a better understanding of the shock wave, flow properties on a slice at $50 \%$ blade height are shown in figure 5. The static pressure is depicted in figure 5(a), which clearly shows the shock wave. A bow shock is present at the leading edge of the blade. The shock wave impinges on the boundary layer on the suction side of the next blade. This causes flow separation, resulting in a low velocity area behind the shock. This is depicted in figure 5(b), which shows the relative Mach number. Figure 5(c) shows that the shock induces transition of the boundary layer, which results in a sudden increase of eddy viscosity. In general the Spalart-Allmaras turbulence model is not capable of predicting transition. Due to the shock, transition is forced at the right position.

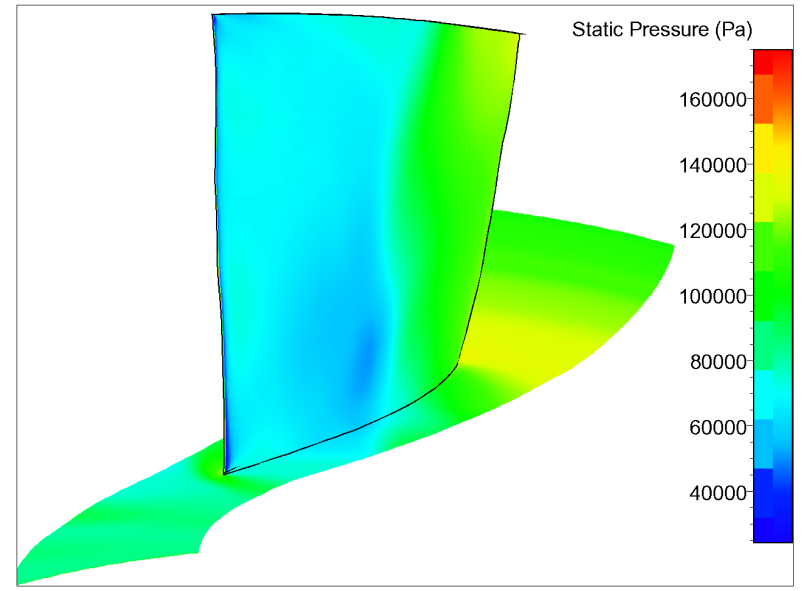

(a)

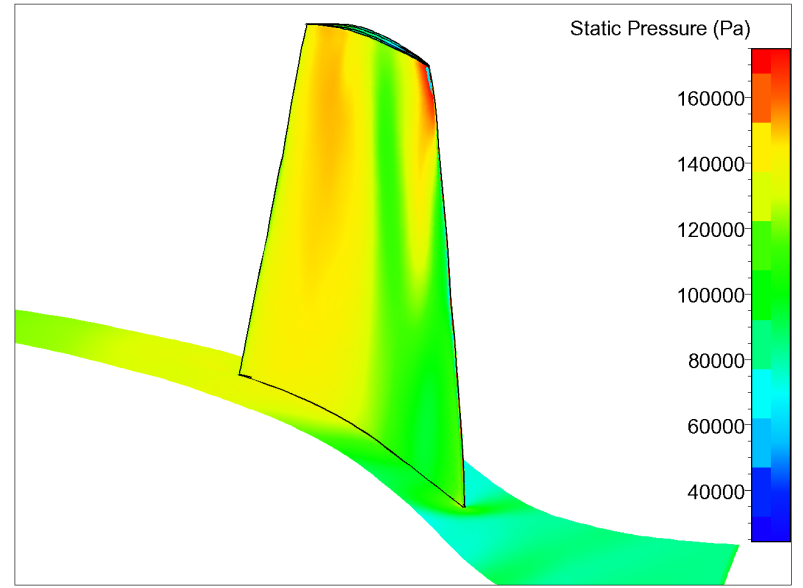

(b)

Figure 4. Static pressure on the surface of the hub at the (a) suction and (b) pressure side of the blade. 


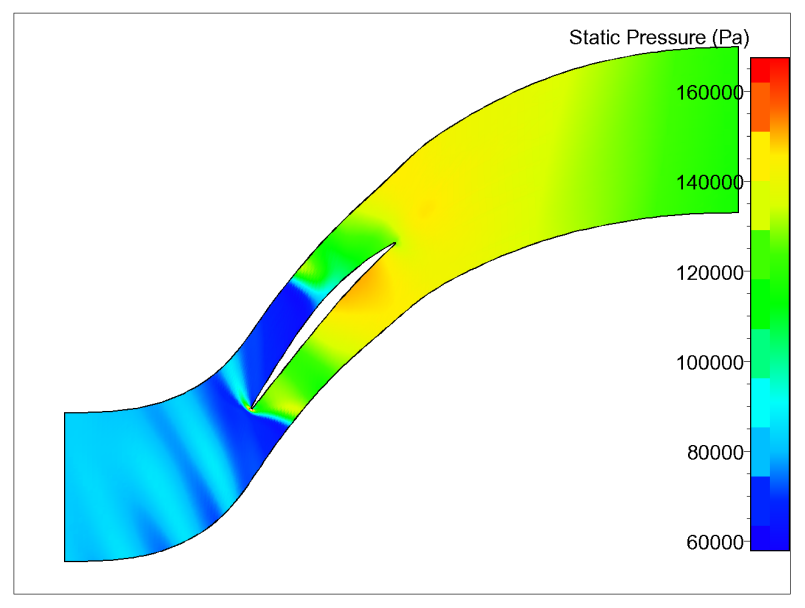

(a)

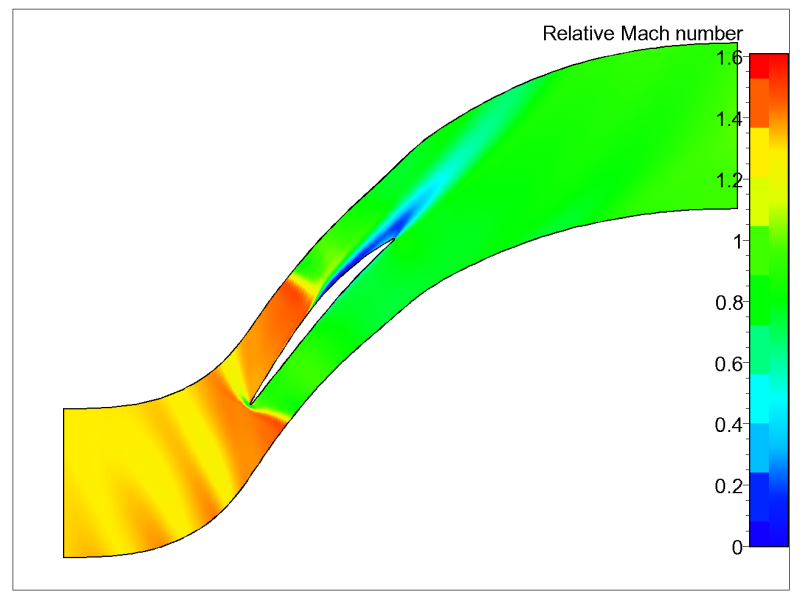

(b)

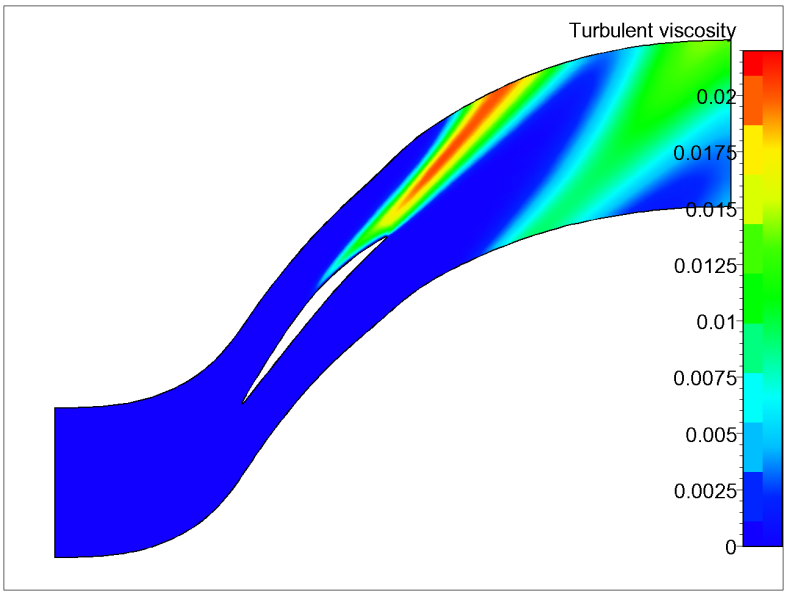

(c)

Figure 5. The static pressure (a), the relative Mach number (b) and the eddy viscosity (c) at $50 \%$ blade height. 


\section{Validation using a quasi-2D simulation}

To validate the Probabilistic Collocation method for NASA Rotor 37, a quasi-2D model is used. The flow contains the characteristics of transonic flow, e.g. a shock wave originating from the leading edge impinging the boundary layer of the next blade. The quasi-2D computation takes about 15 minutes computing time, which enables validation using a Monte Carlo simulation. Based on the Monte Carlo simulation it is concluded that the Probabilistic Collocation method is capable of efficiently propagating the uncertainties to obtain the statistics of the output of interest, like the efficiency of the rotor.

\section{Quasi-2D model}

The quasi-2D model represents a slice at $50 \%$ of the blade height. A block structured grid of 5120 cells is used, it is shown in figure 6(a). It is quasi-2D since it is curved (see figure 6(b)) such that 36 blades fill up a cylinder.

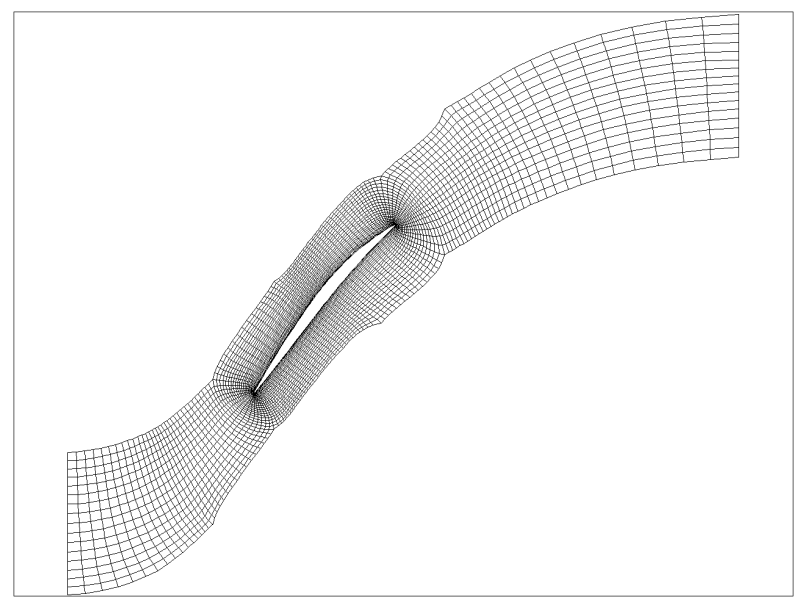

(a)

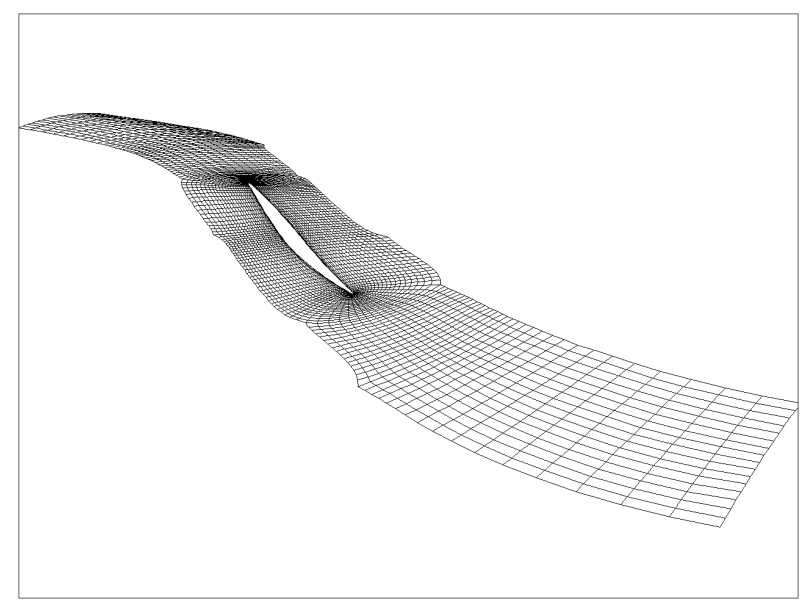

(b)

Figure 6. Quasi 2D computational grid shown in (a) a top view and (b) a 3D view.

The deterministic static pressure and relative Mach number are shown in figure 7. Several blades are put together to make the shock structure more clear. The static pressure (see figure 7(a)) and relative Mach number (see figure 7(b)) compare very well with the true 3D results depicted in figure 5 . The shock wave originates from the leading edge and causes a low speed region behind the shock on the suction side of the next blade.

\section{A. Uncertain static pressure at the outlet}

For the quasi-2D case, an uncertain static outlet pressure $p_{\text {out }}$ is used for validation of the Probabilistic Collocation method. A symmetric beta distribution is used for $p_{\text {out }}$, with parameter settings $\alpha=\beta=4$. This results in a distribution on the interval $[a, b]$ with the maximum probability at the mean/median, i.e. the deterministic value in this case $p_{\text {out }}=1.1$ bar. The interval is chosen to be $\mu_{p_{\text {out }}} \pm 4 \%$. The distribution is shown in figure 8.

\section{Convergence of the Probabilistic Collocation method}

The convergence of the estimated error of the adiabatic efficiency $\eta$, total pressure ratio $p_{2} / p_{1}$, and mass flow $\dot{m}$ with respect to the polynomial order $p$ are shown in figure 9 . The error is estimated using the approximation of one order higher. ${ }^{11}$ For $p=4$, the estimated error has dropped over 2 orders of magnitude. Therefore, a fourth order Probabilistic Collocation approximation is used for further results in this section. 


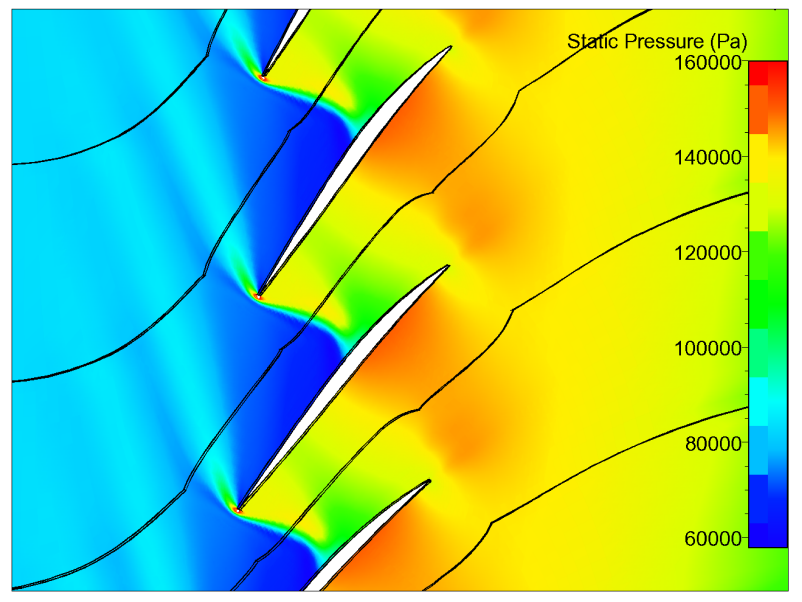

(a)

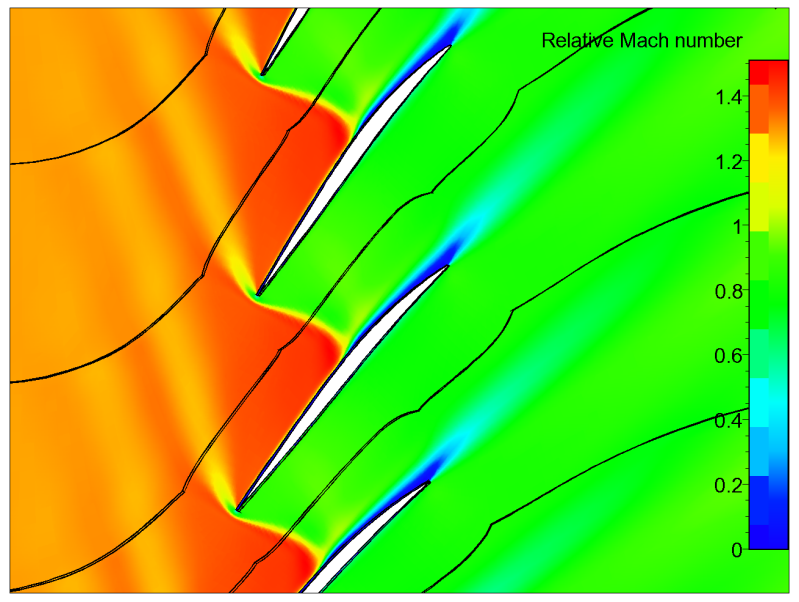

(b)

Figure 7. Quasi-2D flow field close to the blades, with (a) the static pressure and (b) the relative Mach number.

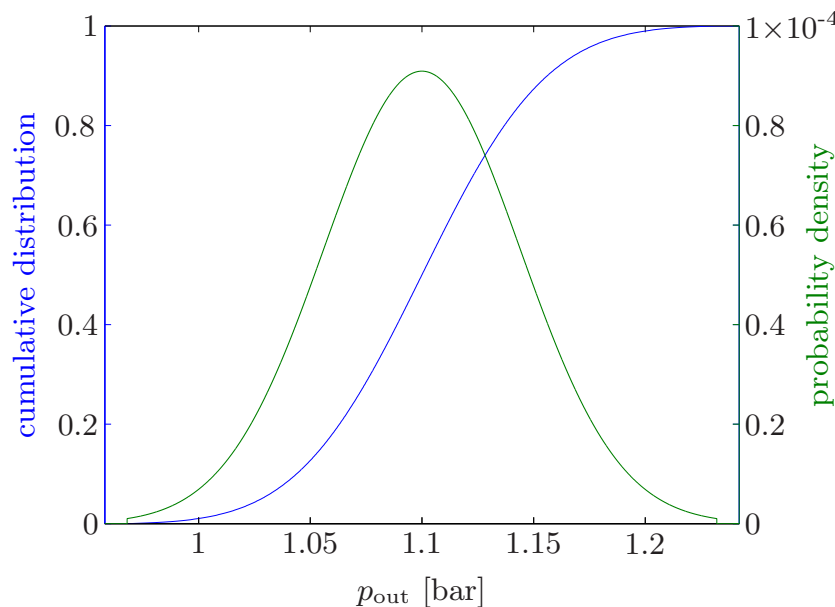

Figure 8. The probability density function (-) and the cumulative distribution function $(-)$ of the static outlet pressure $p_{\text {out }}$. 


\section{B. Stochastic results for the quasi $2 \mathrm{D}$ rotor}

First the validation of the Probabilistic Collocation method with a Monte Carlo simulation is shown for an uncertain static outlet pressure $p_{\text {out }}$. This is done by comparing the distribution functions of $\eta, p_{2} / p_{1}$, and $\dot{m}$, respectively. Secondly, the mean and standard deviation of the pressure and relative Mach number in the flow field are shown.

\section{Comparison with Monte Carlo simulation}

Figures 10-12 show the probability density function as a histogram and the cumulative distribution function of $\eta, p_{2} / p_{1}$, and $\dot{m}$. For the Monte Carlo simulation 10,000 Latin Hypercube samples were used, whereas the Probabilistic Collocation approximation is constructed using 5 deterministic solves (fourth order). The total pressure ratio (figure 10) is almost linearly dependent on the uncertain $p_{\text {out }}$. Therefore, the distribution is close to a beta distribution, similar to the distribution of $p_{\text {out }}$. The efficiency and the mass flow, however, depend nonlinearly on the uncertain $p_{\text {out }}$, as can be seen in figures 11 and 12 respectively. Both quantities obtain a maximum for a certain $p_{\text {out }}$. This results in a distribution with the largest probability near the maximum value. The resuls show good correspondence between the Monte Carlo simulation and the Probabilistic Collocation results
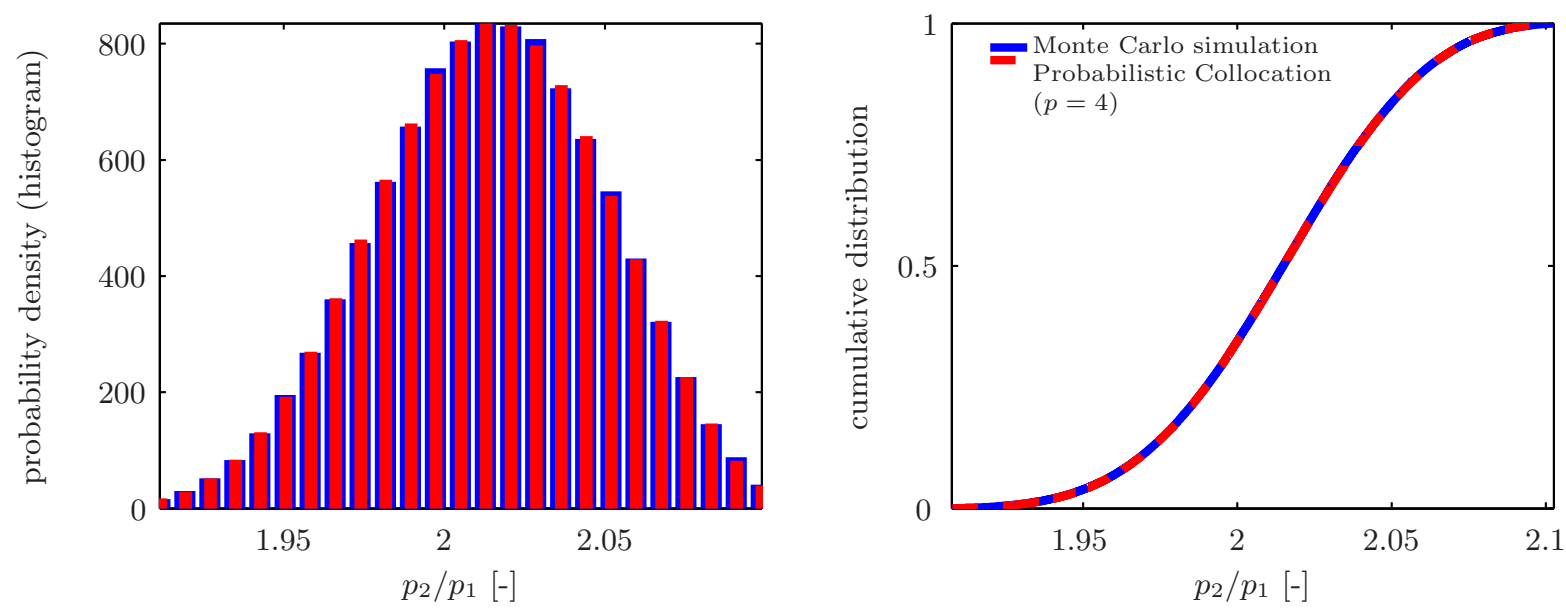

Figure 10. Probability density function (histogram) and cumulative distribution function of the total pressure ratio $p_{2} / p_{1}$ obtained from Monte Carlo simulation (-) using 10,000 Latin Hypercube samples and a fourth order Probabilistic Collocation approximation (--) using 5 deterministic solves.
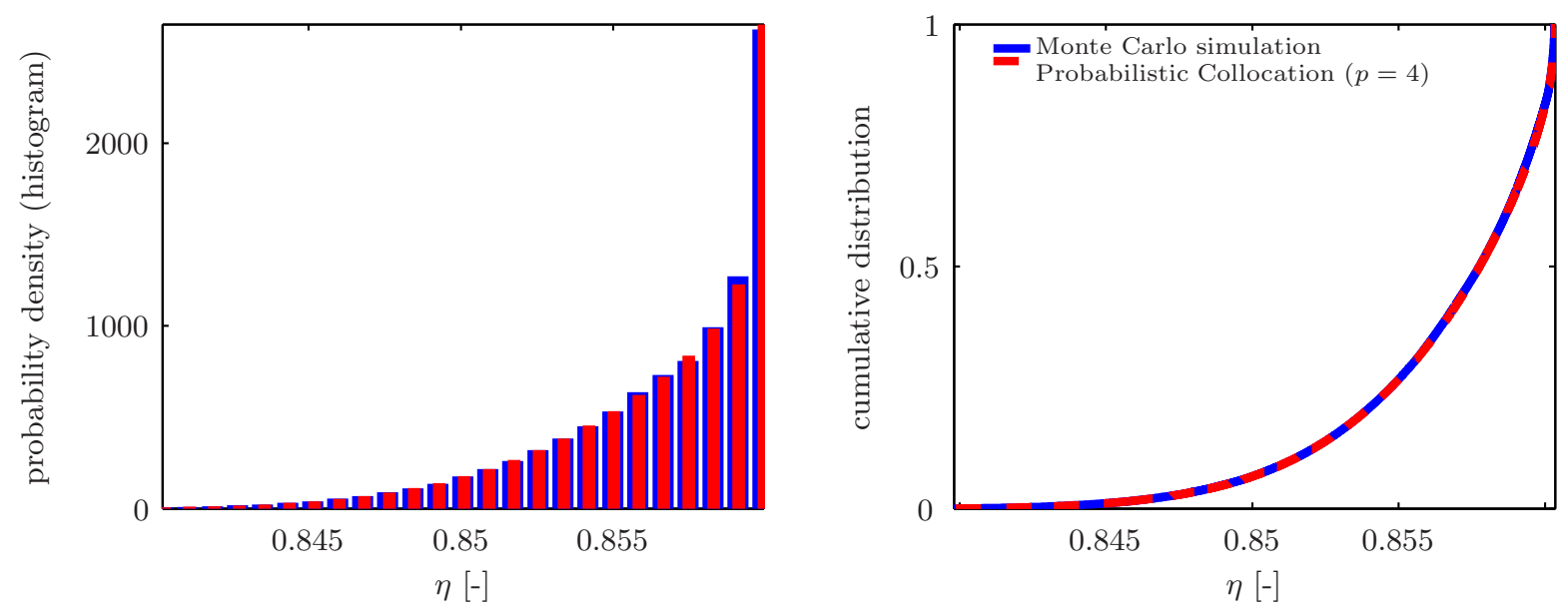

Figure 11. Probability density function (histogram) and cumulative distribution function of the adiabatic efficiency $\eta$ obtained from Monte Carlo simulation (-) using 10,000 Latin Hypercube samples and a fourth order Probabilistic Collocation approximation (--) using 5 deterministic solves. 

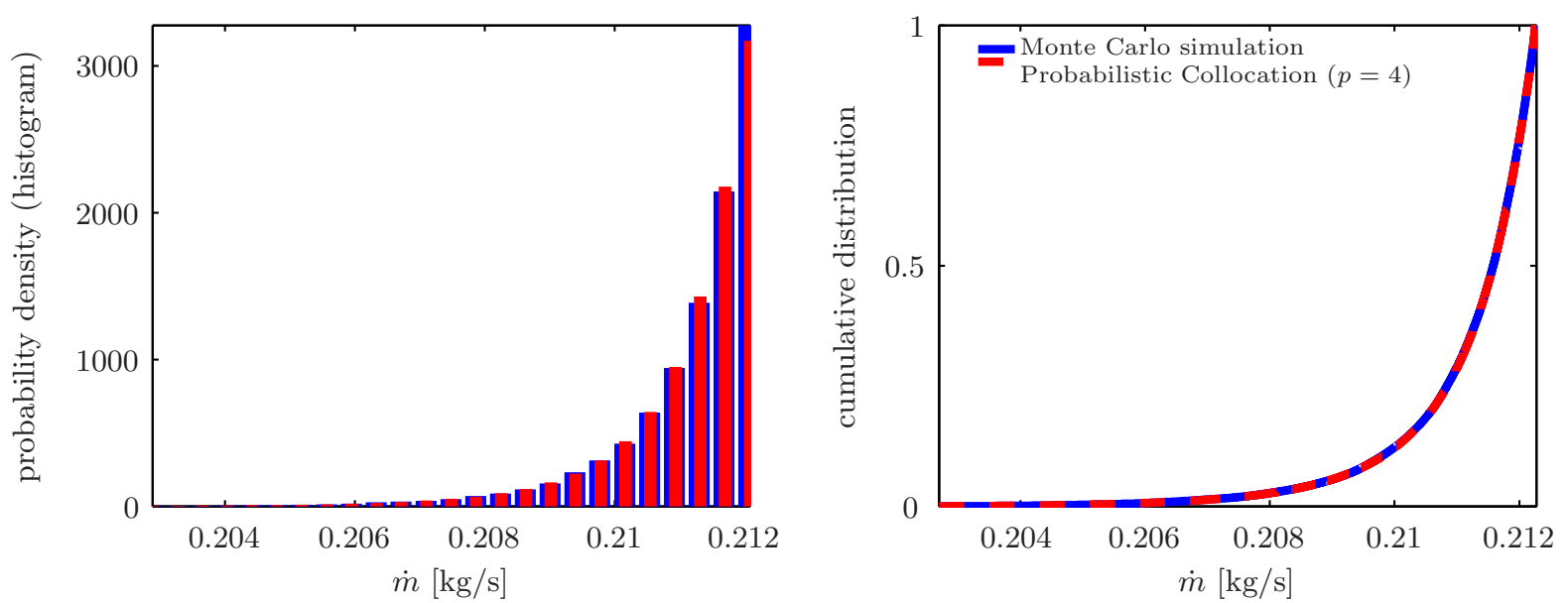

Figure 12. Probability density function (histogram) and cumulative distribution function of the mass flow $\dot{m}$ obtained from Monte Carlo simulation (-) using 10,000 Latin Hypercube samples and a fourth order Probabilistic Collocation approximation (--) using 5 deterministic solves.

\section{Statistics of the flow field}

Figure 13 shows the mean (a) and the standard deviation (b) of the static pressure field close to the blades. The mean is similar to the deterministic solution shown in figure 7(a). The standard deviation (figure 13(b)) shows that due to the uncertain static outlet pressure, largest standard deviation is present inside the shock wave. The largest standard deviation is $26,000 \mathrm{~Pa}$, which corresponds to a local coefficient of variation in the order of $25 \%$.

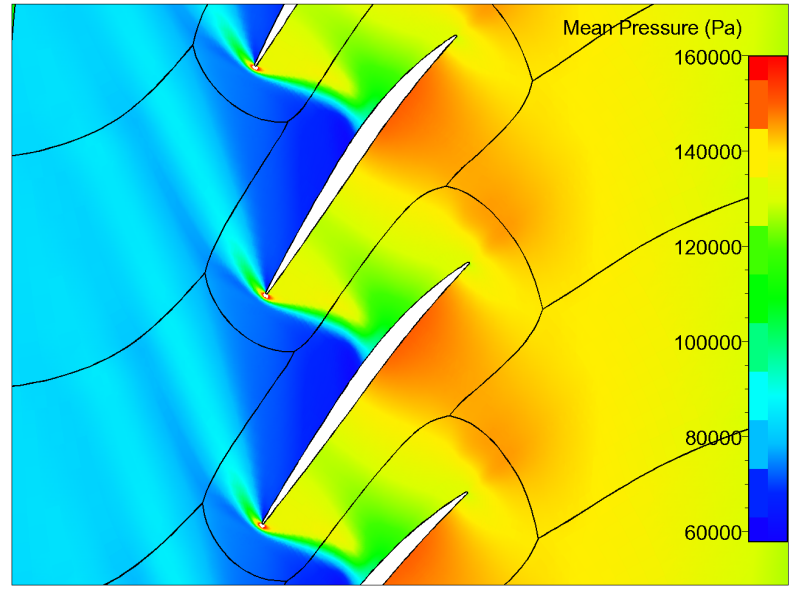

(a)

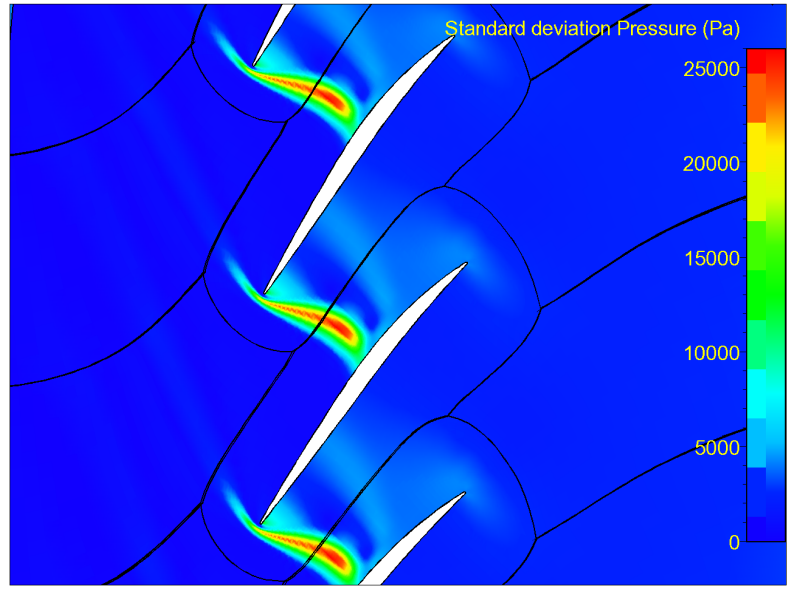

(b)

Figure 13. Quasi-2D static pressure fields close to the rotor blades, with (a) the mean and (b) the standard deviation obtained from a fourth order Probabilistic Collocation approximation, based on an uncertain static outlet pressure.

Figure 14 depicts the mean and standard deviation of the relative Mach number, which shows a similar picture as the static pressure. Again a local coefficient of variation in the order of $25 \%$ is obtained near the shock wave. The difference is near the location where the shock wave interacts with the boundary layer on the suction side of the blade. Here the standard deviation has a peak of 0.26 . 


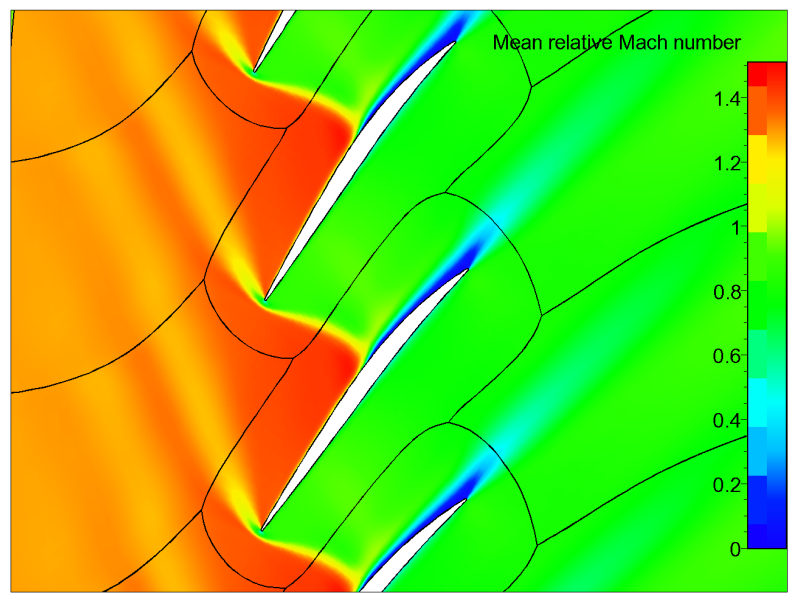

(a)

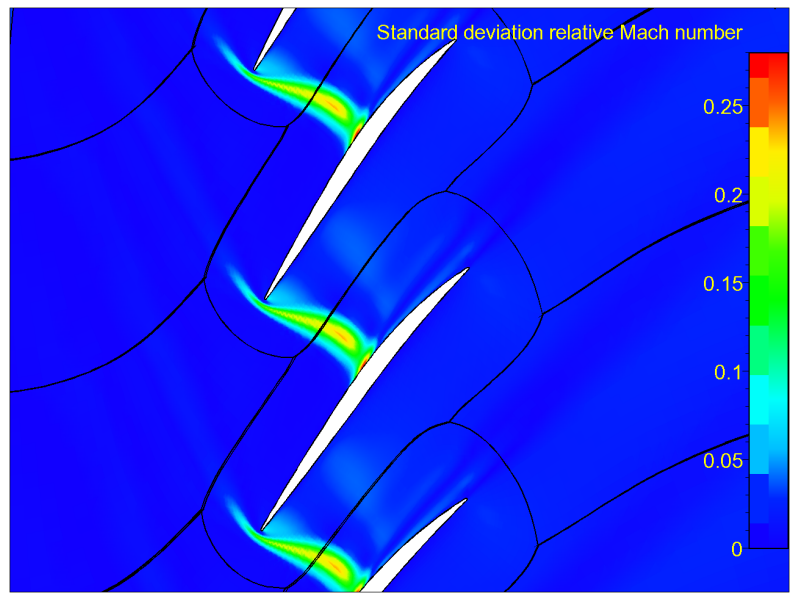

(b)

Figure 14. Quasi-2D relative Mach number fields close to the rotor blades, with (a) the mean and (b) the standard deviation obtained from a fourth order Probabilistic Collocation approximation, based on an uncertain static outlet pressure.

\section{Conclusions of the validation}

Based on the presented results, it can be concluded that the Probabilistic Collocation method is capable of propagating an uncertainty accurately and efficiently. The quantities of interest like adiabatic efficiency, total pressure ratio and mass flow show good correspondence with a Monte Carlo simulation using 10,000 Latin hypercube samples using a fourth order Probabilistic Collocation approximation (requiring only 5 deterministic solves).

The flow fields show that the mean solutions are similar to the deterministic solution. The standard deviation indicates that the solutions are sensitive near the shock wave and the shock wave boundary layer interaction on the suction side of the blades. Local coefficients of variation in the order of $25 \%$ are observed while the coefficient of variation of the uncertain static outlet pressure was only $4 \%$.

\section{NASA Rotor 37, 3D simulations}

After the successful validation of the Probabilistic Collocation method using a quasi-2D setup, it is applied to the 3D simulations of NASA Rotor 37.

\section{A. Uncertain total pressure profile at the inlet}

The second source of uncertainty is the total pressure profile at the inlet of the rotor. According to Reid and Moore ${ }^{3}$ the uncertainty of the measurements of the profile is $\pm 100 \mathrm{~Pa}$. Figure 15 shows the total pressure profile at the inlet, where the blue line indicates the mean/deterministic profile. The profile is assumed to vary $\pm 1 \%$ around the deterministic value, the minimum and maximum pressure profiles are indicated by the red lines in figure 15. This corresponds to a standard deviation of approximately $\sigma_{p} \approx 300 \mathrm{~Pa}$, which is in the same order of the uncertainty of the measurement. The green lines in figure 15 show the profile one standard deviation away from the mean. Again a beta distribution is used with parameter $\alpha=\beta=4$. This results in a symmetric distribution with the mean value as the most probable value. The interval is fixed to $\mu \pm 1 \%$, the coefficient of variation is $C V_{p_{\text {total }, \text { in }}}=0.33 \%$.

\section{B. Stochastic results for the 3D simulations}

Figure 16 shows the convergence of the Probabilistic Collocation method for the 3D simulations of NASA rotor 37 with uncertain total pressure profile at the inlet. The convergence is shown for the adiabatic efficiency, total pressure ratio and mass flow. A second order approximation results in a decrease of the 


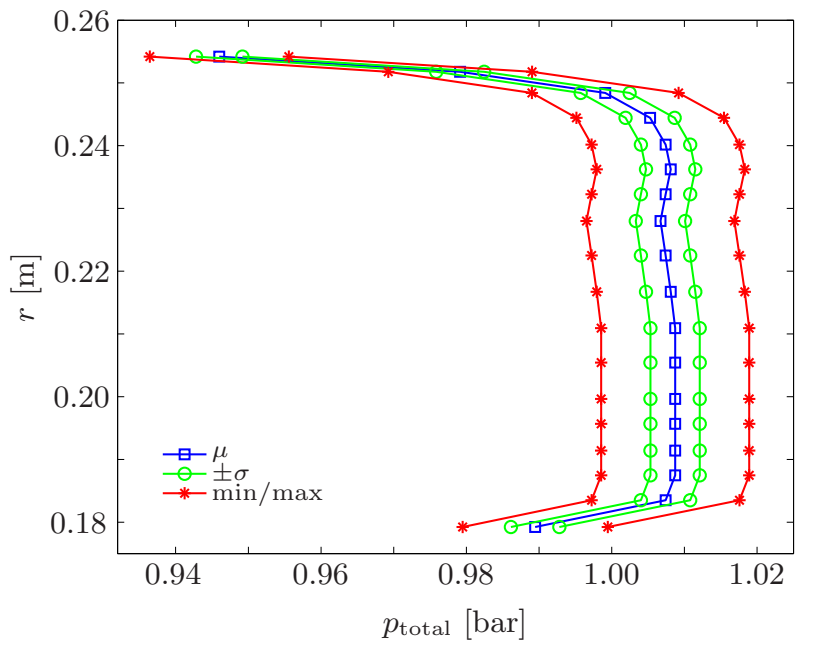

Figure 15. Total pressure profile at the inlet of the rotor. Shown are the mean profile $(\mu), \pm$ one standard deviation $( \pm \sigma)$ and the boundaries of the interval of $\pm 1 \%(\min / \max )$.

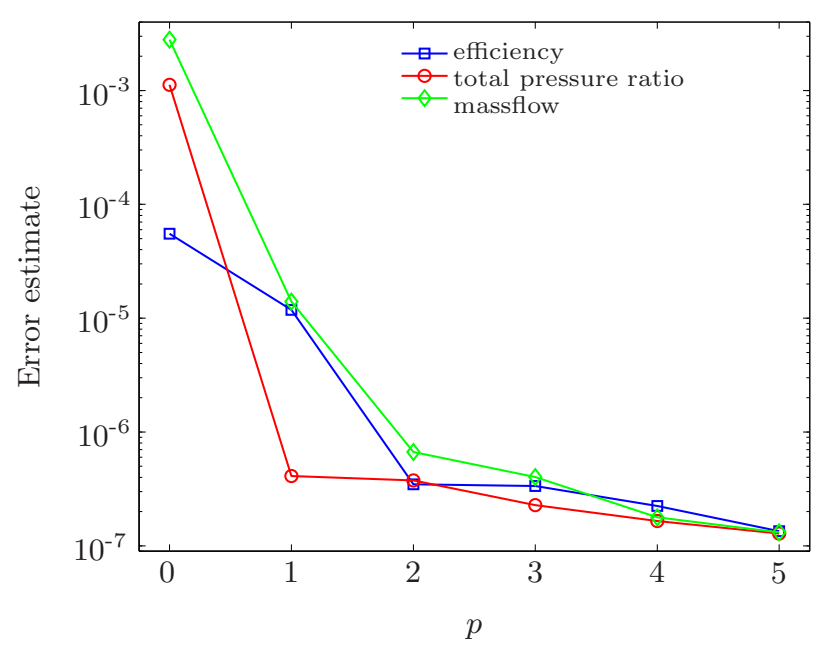

Figure 16. Convergence of the Probabilistic Collocation method for the adiabatic efficiency $\eta$, total pressure ratio $p_{2} / p_{1}$, and mass flow $\dot{m}$ with respect to the polynomial chaos order $p$.

estimated error of more than 2 orders of magnitude. The mean and variance are converged to 8 and 4 significant digits respectively. The probability distribution functions show no difference between the second and higher orders, therefore the second order is used in the remainder of this chapter.

Table 2 summarizes the statistics of $\eta, p_{2} / p_{1}$, and $\dot{m}$ at maximum efficiency. Shown are the mean, standard deviation, the coefficient of variation and the amplification factor. The amplification factor was defined as $\Upsilon=C V_{\text {solution }} / C V_{\text {unc. parameter }}$, the ratio between the $C V$ of the solution and the $C V$ of the uncertain parameter. It is an indication of the sensitivity of the solution with respect to the uncertain parameter. The mean values of the solution are the same as the deterministically found values in table 1. The coefficients of variation are significantly different for each parameter. The adiabatic efficiency shows almost no variation, only $0.008 \%$ and is therefore not sensitive to variations in the total pressure profile at the inlet of the rotor. The amplification factor $\Upsilon_{\eta}$ is 0.024 , which indicates that the uncertainty in the total pressure profile is strongly damped for the efficiency. Secondly, $p_{2} / p_{1}$ shows more variability with a $C V_{p_{2} / p_{1}}$ of $0.158 \%$. The coefficient of variation of the input uncertainty is, however, still damped since the amplification factor $\Upsilon_{p_{2} / p_{1}}=0.48$ is less than 1. The third parameter of interest, $\dot{m}$, shows a $C V_{\dot{m}}$ of $0.386 \%$, which corresponds with an $\Upsilon_{\dot{m}}$ of 1.16. This means the uncertainty of the total pressure profile is amplified for the mass flow. The mass flow is the most sensitive to uncertainties in the total pressure profile at the inlet for this case. This observation agrees with results of Gopinathrao, Bagshaw, Mabilat and Alizadeh. ${ }^{2}$

Figure 17 shows the probability density and cumulative distribution function of $\eta, p_{2} / p_{1}$, and $\dot{m}$. For the current case settings, the solution is not showing strong nonlinear behavior for $p_{2} / p_{1}$ and $\dot{m}$. The rotor is near the flow conditions of maximum efficiency. This results in a nonlinear propagation of the uncertain total pressure profile to the adiabatic efficiency. The distribution of $\eta$ shows the highest probability at the maximum value of $\eta$. The distributions of $p_{2} / p_{1}$ and $\dot{m}$ are close to the beta distribution with $\alpha=\beta=4$, which was the type of distribution of the uncertain total pressure profile at the inlet. It can be seen that, although the standard deviations are small, the intervals of $p_{2} / p_{1}$ and $\dot{m}$ are worth to be taken into account. They cover a range of about $6 \sigma$, which corresponds with $0.95 \%$ and $2.3 \%$ of the mean for $p_{2} / p_{1}$ and $\dot{m}$, respectively.

Figure 18 shows the compressor maps for the mean values of $\dot{m}, \eta$ and $p_{2} / p_{1}$ with uncertainty bars indicating $\pm \sigma$. Table 2 indicated that $\dot{m}$ shows the largest $C V$. This can be seen in the compressor maps, since the uncertainty bars on $\eta$ and $p_{2} / p_{1}$ are barely visible. The bars indicating the standard deviation of $\dot{m}$ are significant. Clearly, it is important to take the uncertainty in the total pressure profile at the inlet into account when compressor maps are computed.

Figure 19 shows the mean and standard deviation of the static pressure in the flow field. Horizontal slices at $25 \%, 50 \%$ and $75 \%$ of the blade height are shown in figure 19(a) and vertical slices at different locations along the hub in figure 19(b) to obtain a picture of the three dimensional features of the flow. The mean 


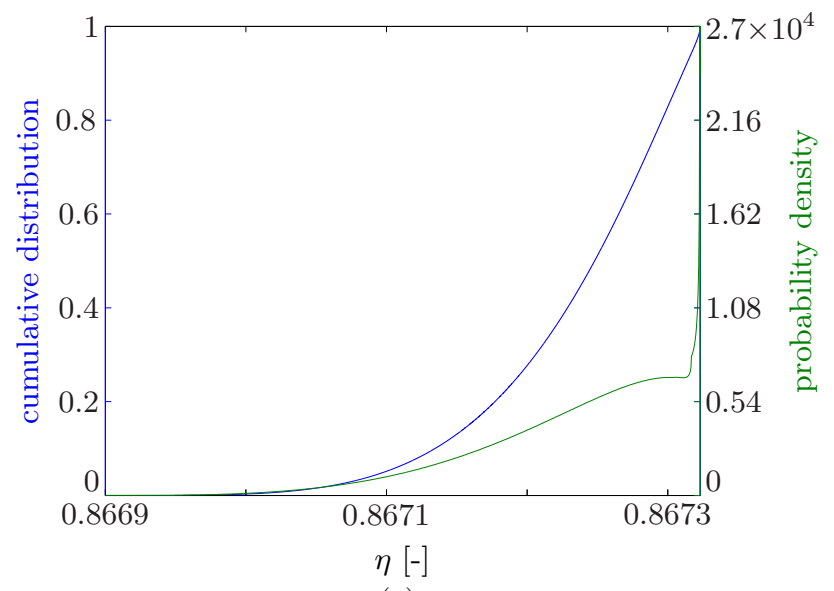

(a)

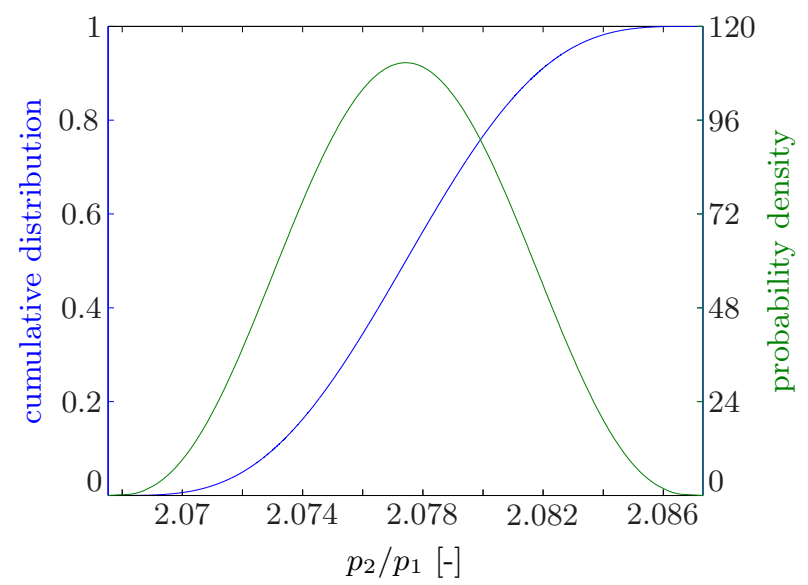

(b)

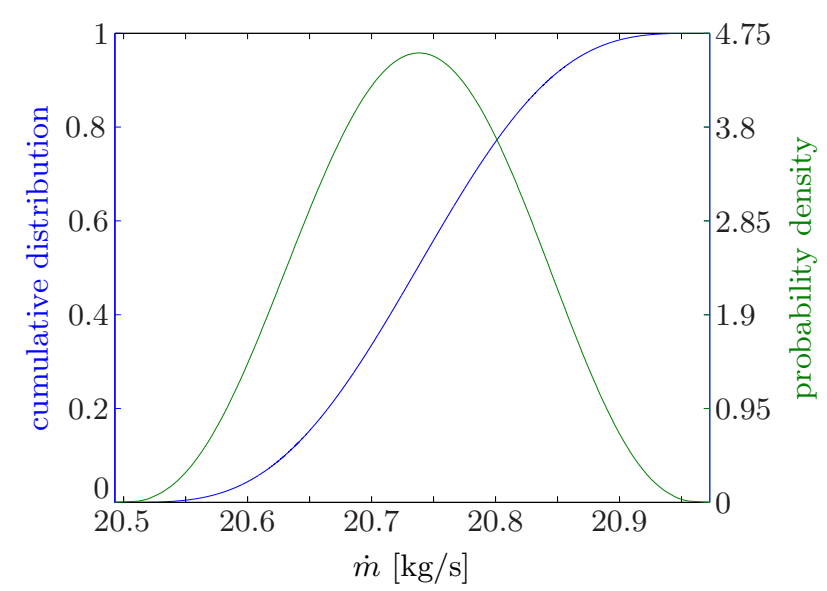

(c)

Figure 17. The probability density function $(-)$ and the cumulative distribution function (-) of the adiabatic efficiency $\eta$, total pressure ratio $p_{2} / p_{1}$ and the mass flow $\dot{m}$, obtained from a second order Probabilistic Collocation approximation for an uncertain total pressure profile at the inlet of the rotor.

Table 2. Statistics of the adiabatic efficiency $\eta$, total pressure ratio $p_{2} / p_{1}$ and mass flow $\dot{m}$ at maximum efficiency of NASA Rotor 37 obtained from a second order Probabilistic Collocation approximation for an uncertain total pressure profile at the inlet of the rotor.

\begin{tabular}{ccccc}
\hline Quantity & $\begin{array}{c}\text { Mean } \\
\boldsymbol{\mu}\end{array}$ & $\begin{array}{c}\text { Standard } \\
\text { deviation } \boldsymbol{\sigma}\end{array}$ & $\begin{array}{c}\text { Coefficient of } \\
\text { variation } \boldsymbol{C} \boldsymbol{V}\end{array}$ & $\begin{array}{c}\text { Amplification } \\
\text { factor } \boldsymbol{\Upsilon}\end{array}$ \\
\hline$\eta$ & 0.867 & 0.000069 & $0.008 \%$ & 0.024 \\
$p_{2} / p_{1}$ & 2.077 & 0.003293 & $0.158 \%$ & 0.48 \\
$\dot{m}$ & 20.737 & 0.08004 & $0.386 \%$ & 1.16 \\
\hline
\end{tabular}




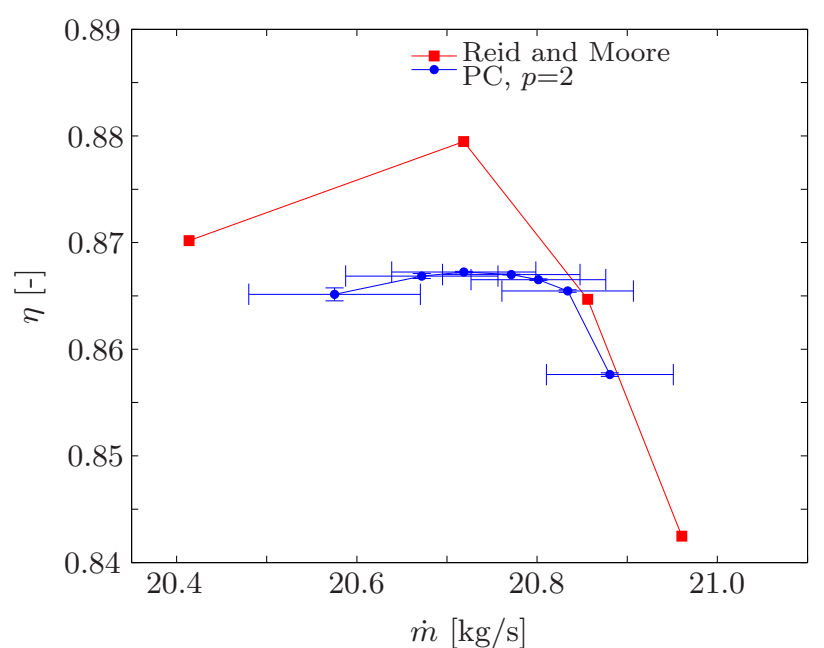

(a)

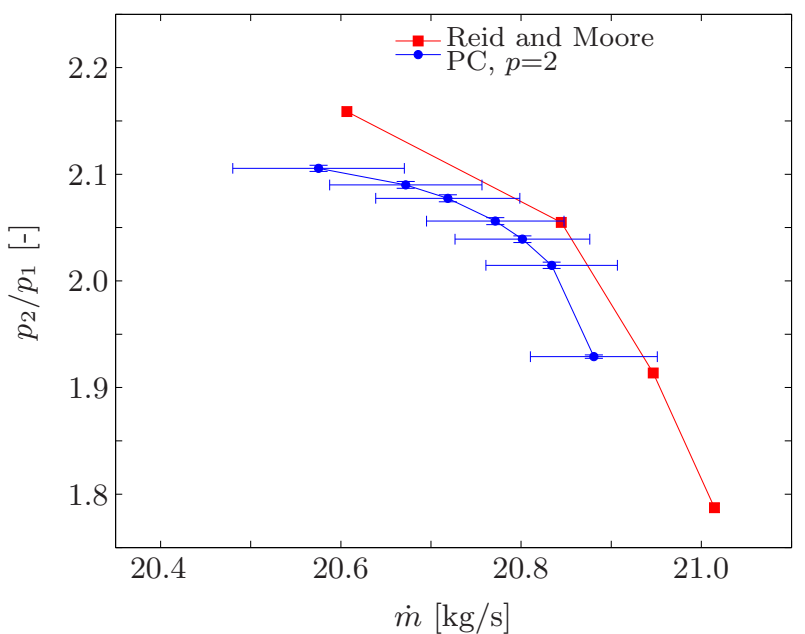

(b)

Figure 18. Compressor maps of NASA Rotor 37, with (a) the adiabatic efficiency $\eta_{\text {adiabatic }}$ and (b) the total pressure ratio $p_{2} / p_{1}$ against the mass flow $\dot{m}$. The figure shows the stochastic results as the mean $\mu$ and uncertainty bars indicating $\pm \sigma$, and the experimental results from Reid and Moore. ${ }^{3}$ Obtained from a second order Probabilistic Collocation approximation for an uncertain total pressure profile at the inlet of the rotor.

static pressure field around the blade is similar to the deterministic simulation. The shock wave becomes stronger near the tip of the blade, due to the higher relative velocity. The largest variation is present in the shock region, as can be seen in figures 19(c) and 19(d).

\section{Conclusions}

Compressor rotors are components of a gas turbine that are highly sensitive to operational and geometrical uncertainties. The Probabilistic Collocation method was used to propagate operational uncertainties through simulations of NASA Rotor 37. Validation of the Probabilistic Collocation method with a Monte Carlo simulation using 10,000 Latin Hypercube samples demonstrated that the Probabilistic Collocation method can successfully be applied to a turbomachinery case. NASA Rotor 37 is a transonic axial flow compressor rotor. The flow is characterized by a bow shock in front of the leading edge. The shock wave impinges on the boundary layer of the next blade, causing separation and transition instantaneously.

The total pressure profile at the inlet of the rotor is assumed to be uncertain. A symmetric beta distribution was used for the pressure profile, with the standard deviation such that the uncertainty is in the same order of the measurement accuracy reported in literature. The mass flow was shown to be the most sensitive to the uncertainty, while the efficiency is least affected. The compressor maps are constructed as functions of the mass flow. It was shown to be important to take the uncertainty in the total pressure profile at the inlet into account. The mean static pressure field around the blade does not differ much from the deterministic simulation. The standard deviation of the static pressure showed that the largest variation is present near the shock wave and mainly in the region of the strongest shock, which is near the tip of the blade.

\section{Acknowledgments}

The presented work is supported by the NODESIM-CFD project (Non-Deterministic Simulation for CFD based design methodologies); a collaborative project funded by the European Commission, Research Directorate-General in the $6^{\text {th }}$ Framework Programme, under contract AST5-CT-2006-030959. 


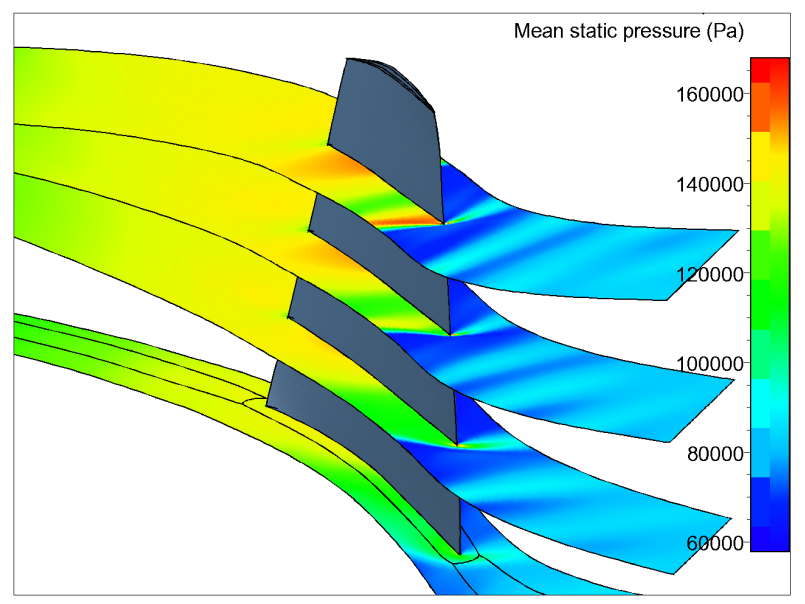

(a)

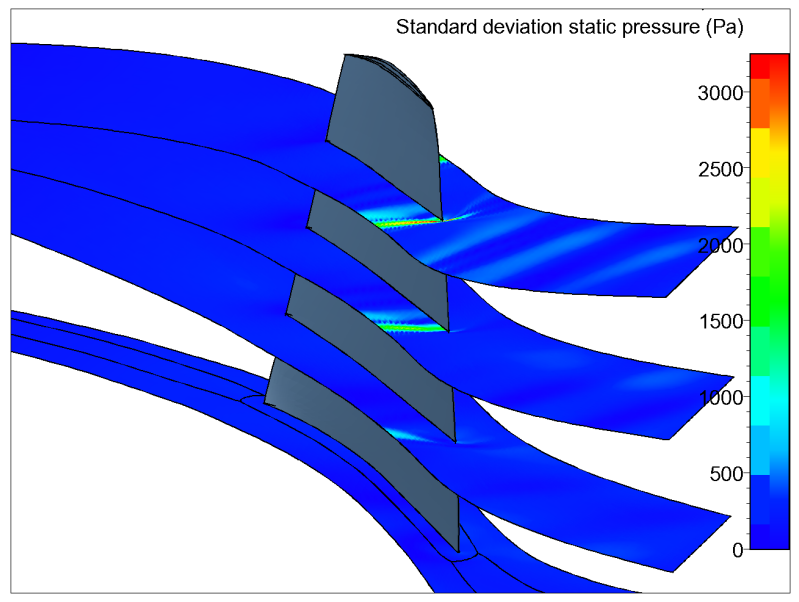

(c)

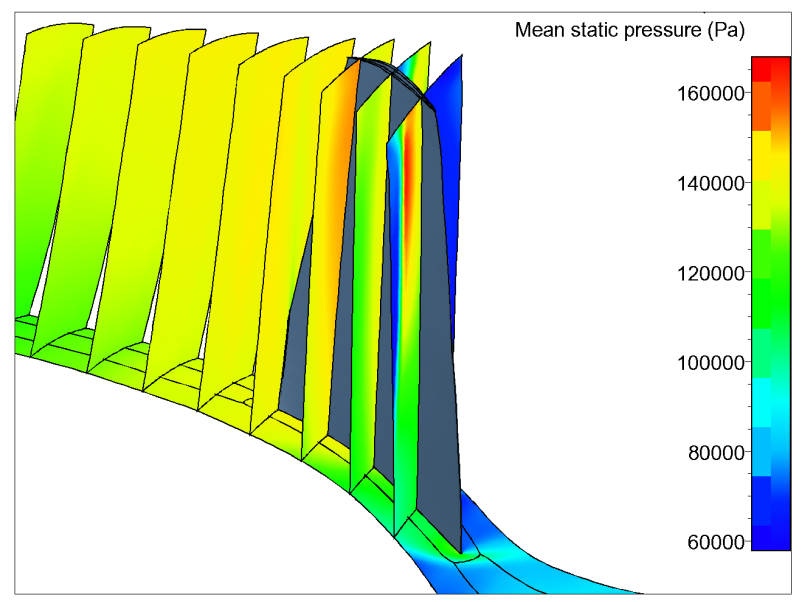

(b)

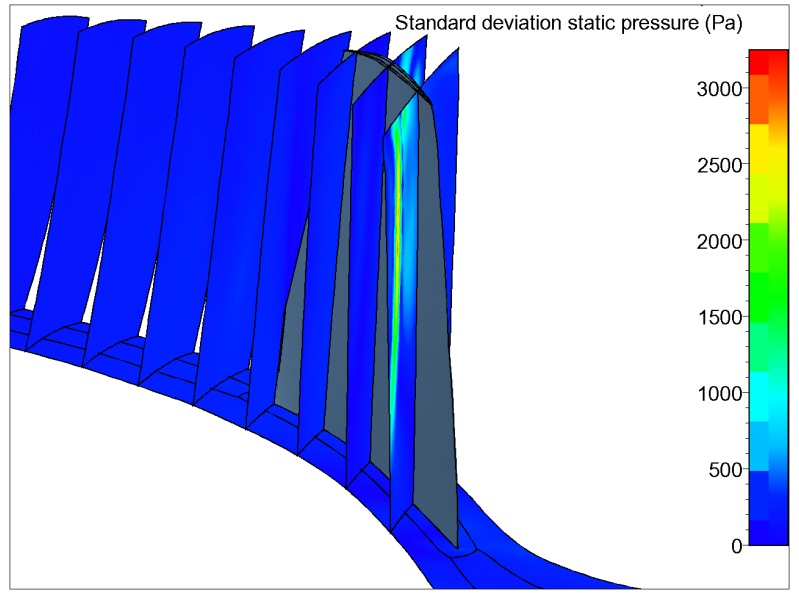

(d)

Figure 19. Static pressure fields (a) and (b) mean and (c) and (d) standard deviation. The horizontal slices are at $\mathbf{2 5 \%}, \mathbf{5 0 \%}$ and $\mathbf{7 5 \%}$ of the blade height, obtained from a second order Probabilistic Collocation approximation for an uncertain total pressure profile at the inlet of the rotor. 


\section{References}

${ }^{1}$ Gopinathrao, N., Mabilat, C., and Alizadeh, S., "Non-Deterministic Thermo-Fluid Analysis of a Compressor Rotor-Stator Cavity," Proceedings of the 50th AIAA/ASME/ASCE/AHS/ASC Structures, Structural Dynamics and Materials Conference, AIAA paper 2009-2278, Schaumburg(IL), United States, May 2009.

${ }^{2}$ Gopinathrao, N., Bagshaw, D., Mabilat, C., and Alizadeh, S., "Non-Deterministic CFD Simulation of a Transonic Compressor Rotor," Proceedings of ASME Turbo Expo 2009: Power for Land, Sea and Air, GT2009-60122, Orlando (FL), United States, June 2009.

${ }^{3}$ Reid, L. and Moore, R. D., "Design and Overall Performance of Four Highly Loaded, High-Speed Inlet Stages for an Advanced High-Pressure-Ratio Core Compressor," Tech. Rep. NASA-TP-1337, NASA Lewis Research Center, 1978.

${ }^{4}$ Dunham, J., "CFD Validation for Propulsion System Components," Tech. Rep. AR-355, AGARD, May 1998.

${ }^{5}$ Wisler, D. C., "CFD Code assessment in turbomachinery - author's information package," unpublished ASME note.

${ }^{6}$ Chima, R., "Calculation of tip clearance effects in a transonic compressor rotor," Journal of Turbomachinery, Vol. 120, No. 1, 1998, pp. 131-140.

${ }^{7}$ Gerolymos, G. and Vallet, I., "Tip-clearance and secondary flows in a transonic compressor rotor," Journal of Turbomachinery, Vol. 121, 1999, pp. 751-762.

${ }^{8}$ Beheshti, B., Teixeira, J., Ivey, P., Ghorbanian, K., and Farhanieh, B., "Parametric study of tip clearance - Casing treatment on performance and stability of a transonic axial compressor," Journal of Turbomachinery, Vol. 126, 2004, pp. 527535.

${ }^{9}$ Babuška, I., Nobile, F., and Tempone, R., "A Stochastic Collocation Method for Elliptic Partial Differential Equations with Random Input Data," SIAM Journal of Numerical Analysis, Vol. 45, No. 3, 2007, pp. 1005-1034.

${ }^{10}$ Loeven, G., Witteveen, J., and Bijl, H., "Probabilistic Collocation: an efficient non-intrusive approach for arbitrarily distributed parametric uncertainties," Proceedings of the 45th AIAA Aerospace Sciences Meeting and Exhibit, AIAA paper 2007-317, Reno(NV), United States, January 2007.

${ }^{11}$ Loeven, G. and Bijl, H., "Probabilistic Collocation used in a Two-Step approach for efficient uncertainty quantification in computational fluid dynamics," CMES Computer Modeling in Engineering \& Sciences, Vol. 36, No. 3, 2008, pp. 193-212.

${ }^{12}$ Loeven, G. and Bijl, H., "Application of the Probabilistic Collocation method to transonic turbulent flow around a RAE2822 airfoil," NODESIM-CFD Workshop on Quantification of CFD Uncertainties, Brussels, Belgium, 2009.

${ }^{13}$ Tartinville, B. and Hirsch, C., "Rotor 37," Notes in Numerical Fluid Mechanics and Multidisciplinary Design, Vol. 94, 2006, pp. 183-202, FLOMANIA - A European Initiative on Flow Physics Modelling. 